JOURNAL CLUB

\title{
Short-term use of clarithromycin in lower respiratory tract infections may increase long-term risk of cardiovascular mortality and morbidity
}

Macrolides are commonly prescribed for chronic obstructive pulmonary disease (COPD) exacerbations and community-acquired pneumonia. The short-term cardiovascular effects, probably due to QT prolongation, are well documented and some studies have implicated macrolides with increased mortality in patients with pre-existing coronary disease.

This study evaluated the association between clarithromycin use and cardiovascular events in patients treated for lower respiratory tract infections in a secondary analysis of two prospective cohort studies. The EXODUS dataset enrolled 1343 patients aged over 40 admitted with COPD exacerbations to 12 hospitals in the UK from 2009 to 2011. The Edinburgh pneumonia cohort included 1631 patients admitted to hospital with radiologically confirmed community-acquired pneumonia from 2005 to 2009. Both datasets were reviewed for cardiovascular events at 1 year (defined as hospital admission for acute coronary syndrome, decompensated cardiac failure, serious arrhythmia or sudden cardiac death). There were 268 events in the COPD cohort and 171 in the pneumonia cohort.

The use of clarithromycin was associated with an increased risk of cardiovascular events at 1 year in both the cohorts after adjustment for confounders, with a HR of 1.5 (95\% CI 1.13 to 1.97) for COPD and 1.68 (1.18 to 2.38) for pneumonia. This remained significant after propensity-adjusted analysis to correct for patients with more severe illness being given clarithromycin. It is of interest to note that the increased risk was only significant in those receiving $\geq 7$ days of clarithromycin, older patients ( $>60$ for COPD, $>70$ for pneumonia) and those with pre-existing cardiovascular disease. The increased risk was not seen in patients with COPD treated with alternative antibiotics ( $\beta$ lactams and doxycycline).

While this study is observational in nature, the results are troubling, particularly given the trend towards prescribing long-term macrolides to prevent COPD exacerbations and further studies need to be performed to explore this potential risk.

- Schembri S, Williamson PA, Short PM, et al. Cardiovascular events after clarithromycin use in lower respiratory tract infections: analysis of two prospective cohort studies. BMJ 2013;346:f1235.

Haider Al-Najjar

Correspondence to Dr Haider Al-Najjar, SPR in Respiratory, Southmead Hospital, Southmead Road, Bristol BS10 5NB, UK; haidernajjar@hotmail.com

Competing interests None.

Provenance and peer review Not commissioned; internally peer reviewed.

To cite Al-Najjar H. Thorax 2014:69:288.

Thorax 2014;69:288. doi:10.1136/thoraxjnl-2013-204080 